\title{
Treatment-induced tumor cell apoptosis following high-intensity interval training and saffron aqueous extract in mice with breast cancer
}

\author{
F. AHMADABADI ${ }^{1}$, M. SAGHEBJOO ${ }^{1}$, M. HEDAYATI ${ }^{2}$, R. $\operatorname{HOSHYAR}^{3}$ and \\ C.-J. HUANG ${ }^{4 *}$
}

${ }^{1}$ Department of Exercise Physiology, Faculty of Sport Sciences, University of Birjand, Birjand, Islamic Republic of Iran

${ }^{2}$ Cellular and Molecular Endocrine Research Center, Research Institute for Endocrine Sciences, Shahid Beheshti University of Medical Sciences, Tehran, Islamic Republic of Iran

${ }^{3}$ Cellular and Molecular Research Center, Birjand University of Medical Sciences, Birjand, Islamic Republic of Iran

${ }^{4}$ Exercise Biochemistry Laboratory, Department of Exercise Science and Health Promotion, Florida Atlantic University, Boca Raton, FL, 33431, USA

Received: September 2, 2020 • Accepted: February 1, 2021

Published online: April 14, 2021

(c) 2021 Akadémiai Kiadó, Budapest

\begin{abstract}
This study was to investigate whether high-intensity interval training (HIIT) and saffron aqueous extract (SAE) would provide a synergistic effect to improve tumor volume reduction and also modulate pro- and anti-apoptotic protein expression in tumor tissue of $4 \mathrm{~T} 1$ breast cancer-bearing mice. Female mice following induction of breast cancer through injection of $4 \mathrm{~T} 1$ cell lines were randomly divided into four groups: (1) HIIT, (2) SAE, (3) HIIT+ SAE, and (4) control. The tumor volume was significantly lower in the HIIT, SAE, and HIIT+SAE groups than in the controls. The protein level of caspase- 3 in the HIIT and the SAE groups was higher than in the control and the HIIT+SAE groups. The Bax protein level in the SAE group was higher than in the control. The HIIT+SAE group showed a lower level of Bax than the HIIT and the SAE groups. The protein level of Bcl-2 was higher in the HIIT+SAE vs. both the HIIT and the SAE groups. Finally, the ratio of $\mathrm{Bax} / \mathrm{Bcl}-2$ was significantly higher in the HIIT and the SAE groups than in the HIIT+SAE and control groups. These findings indicate that a combination of HIIT and SAE interventions does not improve the apoptotic induction in tumor tissue, while both HIIT and SAE treatments may
\end{abstract}

* Corresponding author. FACSM 777 Glades Road, FH11A-126B, Boca Raton, Florida, 33431, USA. Tel. +1 561 297 1271. E-mail: chuang5@fau.edu 
mediate apoptotic pathway as evinced by the elevated ratio of Bax/Bcl-2 and caspase-3 levels during tumor progression in breast cancer-bearing mice.

\section{KEYWORDS}

exercise, high-intensity interval training, Crocus Sativus L. extract, caspase-3, Bax/Bcl-2, apoptosis, breast cancer

\section{INTRODUCTION}

The worldwide prevalence of female breast cancer remains elevated with clinical challenges for prevention [1]. Research has demonstrated that exercise training and nutritional interventions could be an effective approach not only to enhance cellular homeostasis [2], but also to minimize tumor cell proliferation of breast cancer [3]. The inhibition of tumor metastasis and normalization of the tissue microenvironment have been observed in human breast tumors following continuous aerobic training (CAT) [4]. Moreover, it is widely accepted that exercising at higher intensities can be safely used as an effective therapeutic intervention in patients with breast cancer, including the reduction of tumor cells and inhibition of tumor growth [2]. In this regard, high-intensity interval training (HIIT) has been shown to mediate the reduction of tumor cells in patient with breast cancer [5].

Nowadays, medicinal plants as antioxidant agents have been shown to prevent and/or treat breast cancer as an alternative approach with minimal side effects [6]. In particular, Crocus sativus L., commonly known as saffron, has anti-cancer and anti-metastatic properties to promote apoptosis in different cancer cell lines [7]. Apoptosis is genetically programmed cell death, which plays an essential role under physiological and pathological conditions; however, this apoptotic process is usually evaded by cancer cells [8]. Two primary apoptotic pathways are identified, operating through either extracellular signals (death receptor pathway) or internal stimuli (mitochondrial pathway), such as DNA damage or oxidative stress. Particularly, members of the B-cell lymphoma 2 (Bcl-2) family proteins serve as critical mediators in the mitochondrial pathway of apoptosis and regulate cellular mechanisms underlying the anti- and pro-apoptotic activities of Bcl-2 [9]. Pro-apoptotic Bcl-2 family molecules, such as the Bcl-2associated $\mathrm{X}$ protein (Bax) induce the release of cytochrome $c$ and consequently cancer cell apoptosis [9]. During the apoptotic processes, protease enzymes, such as caspases (e.g., caspase3) are involved in programmed cell death through both pathways in breast cancer patients [10].

Although treatment with antioxidant supplements, such as crocin (a saffron derivative) has been shown to enhance apoptosis with increased ratio of Bax/Bcl-2 and activation of caspases in human gastric adenocarcinoma cells [11], the potential beneficial effects of combined HIIT and saffron on apoptosis in breast tumor tissue have not been investigated. Therefore, this study was to explore whether or not a time-efficient exercise, HIIT and saffron aqueous extract (SAE) supplementation would provide a synergistic effect to reduce tumor volume, along with modulation of pro- and anti-apoptotic protein expression (caspase-3, Bax, and Bcl-2) in tumor tissue of $4 \mathrm{~T} 1$ breast cancer-bearing mice. 


\section{MATERIALS AND METHODS}

\section{Animals}

Female BALB/c mice, weighting $16.1 \pm 1.76 \mathrm{~g}$ and $4-5$ weeks old, were housed under standard laboratory conditions (at $22 \pm 2{ }^{\circ} \mathrm{C}$ with regular 12-h light 12-h dark cycles) and fed a standard laboratory diet and water ad libitum. Subsequently, mice were randomly distributed into four groups ( $N=10$ /group): (1) control, (2) HIIT, (3) SAE, (4) HIIT+ SAE following approximately 7-10 days of tumor injection with observation of a solid tumor. If tumors were not detected during this period, then the mice were considered as non-cancerous. Three mice in the control group, two mice in each of the HIIT+SAE and SAE groups, and one mouse in the HIIT group died during the experiment. The use and care of animals followed the guidelines of the Iranian Convention for the Protection of Vertebrate Animals Used for Experimental and other Scientific Purposes, and authorized by the Ethics Committee of Birjand University of Medical Sciences, Birjand, Iran (IR.BUMS.REC.1397.38).

\section{Cell culture}

A highly metastatic breast cancer cell line, 4T1, derived from a spontaneously arising BALB/c mammary tumor was purchased from the National Cell Bank of Iran, Tehran, Iran. The 4T1 cells were maintained in monolayer cultures in Dulbecco's modified Eagle's medium (DMEM) supplemented with $10 \%$ fetal bovine serum (FBS) at $37{ }^{\circ} \mathrm{C}$ in $5 \% \mathrm{CO}_{2}$. Subsequently, the $4 \mathrm{~T} 1$ cells were resuspended at $1 \times 10^{6}$ cells in $0.2 \mathrm{~mL}$ phosphate buffered saline (PBS) and subcutaneously injected in the left flank of $\mathrm{BALB} / \mathrm{c}$ mice. The respective treatments started after the tumors were observed.

\section{Experimental protocols}

Following familiarization over 3 days with $15 \mathrm{~min}$ of treadmill-running at $10-15 \mathrm{~m} / \mathrm{min}$, mice performed an exercise capacity test every week of training. The HIIT protocol included treadmill running, consisting of six intervals $\left(3 \mathrm{~min}\right.$ and $20 \mathrm{~s}$ at $80-95 \% \mathrm{VO}_{2 \max }$ with one-minute rest between each interval at 30-35\% $\mathrm{VO}_{2 \max }$ for $35 \mathrm{~min} 5 \mathrm{~d}$ /week for 4 weeks). Five-minute warm-up and cool-down at $30-40 \% \mathrm{VO}_{2 \max }$ were also included. The control group was subjected to the noise of the treadmill during the period when mice of the other groups were trained, was housed near the treadmill without food, water (during the time of the exercise bouts), and did not exercise.

Mice in the SAE and HIIT+SAE treatment groups orally consumed $200 \mathrm{mg}$ of saffron per kilogram $(\mathrm{kg})$ of their body weight, with the frequency of three days/week for 4 weeks. The control group was treated with normal saline three times each week during the experimental period. The dose of saffron was selected based on a previously reported pharmacological study of this plant [12].

\section{Measurement of tumor volume}

Tumors were sized in two dimensions. The larger tumor dimension was considered as length (L), and the other $\left(\right.$ at $90^{\circ}$ ) as width $(\mathrm{W})$. After appearance of the tumor, the length and width of the tumor were sized by a digital caliper (STAINLESS HARDENED, LOMBARD, Poland, range 0-150 mm) twice a week. The tumor volume was measured by the formula $\left[\mathrm{V}=1 / 2\left(\mathrm{~L} \times \mathrm{W}^{2}\right)\right]$, according to a previously published study [13]. 


\section{Tissue sample preparation and determination of apoptotic indices}

To avoid acute exercise effects, mice were euthanized $48 \mathrm{~h}$ after the last intervention session by intraperitoneal injection of ketamine $(90 \mathrm{mg} / \mathrm{kg})$ and xylazine $(10 \mathrm{mg} / \mathrm{kg})$. After the sacrifice, the tumor tissues were quickly removed and frozen in liquid nitrogen $\left(-196^{\circ} \mathrm{C}\right)$, then stored at $-80{ }^{\circ} \mathrm{C}$ for further analyses. After the tissue samples were homogenized in suitable buffer (PBS, pH 7.4, 100 $\mathrm{mM})$ and centrifuged (10,000 RPM for $10 \mathrm{~min})$, the supernatants were carefully collected and quantified for total protein levels using a Bradford protein assay kit (Colorimetric, ZellBio GmbH, Ulm, Germany). Afterwards, the protein levels of caspase-3, Bcl-2, and Bax were determined using the appropriate enzyme-linked immunosorbent assay (ELISA) kits (ZellBio GmbH, Ulm, Germany).

\section{Statistical analysis}

All statistical analyses were performed using SPSS version 20.0 (SPSS Inc., Chicago, IL, USA). Given normal distributions, a two-way analysis of variance (ANOVA) with repeated measures (group $\times$ time) with Bonferroni's post hoc test was used to examine the differences in tumor volume. One-way ANOVA and LSD post hoc test were used to determine the significance of differences between groups for apoptotic indices (Caspase-3, Bax, and Bcl-2). Significant differences were defined as $P \leq 0.05$. Data are presented as mean \pm standard deviation (SD).

\section{RESULTS}

\section{Tumor volume}

A significant group-by-time interaction in tumor volume was observed $(\mathrm{F}[21,161)=4.60, P=$ 0.01]. Specifically, the tumor volume incline growth in the HIIT, SAE, and HIIT+SAE groups was lower than that in the control group on day 28 (Fig. 1).

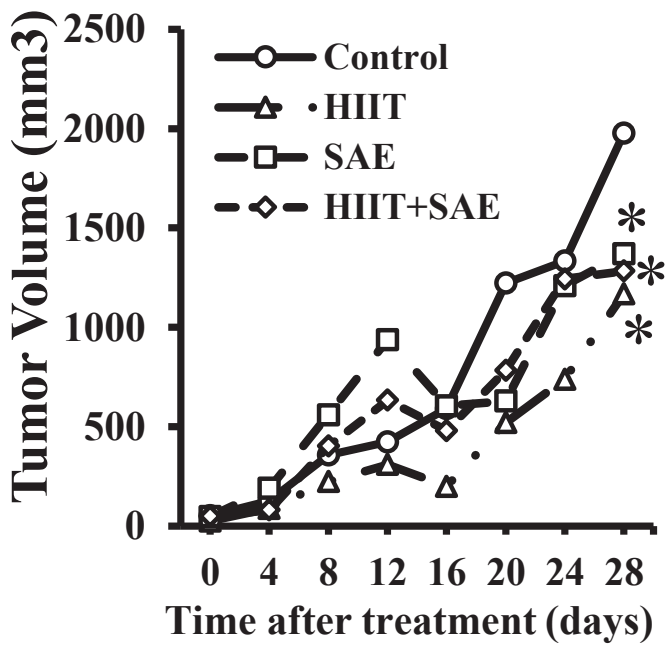

Fig. 1. Effects of HIIT and SAE treatment on the tumor volume of BALB/c mice as a function of time.

* Significant difference vs. the control group. HIIT: high-intensity interval training; SAE: saffron aqueous extract 


\section{The expression of apoptotic indices: caspase-3, Bax and Bcl-2 in tumor tissue}

The level of caspase-3 protein in the HIIT and SAE groups was significantly higher than in the control group $(\mathrm{F}[3,28]=6.16, P=0.05$ and $P<0.01)$ (Fig. 2A). Furthermore, the combined HIIT+SAE group showed a lower level of caspase- 3 than either the HIIT or the SAE group $(P=$ 0.02 and $P<0.01$, respectively) (Fig. 2A). However, no significant difference was found between the HIIT and the SAE-treated groups.

The expression of Bax protein in the SAE group was higher than in the control group (F [3, $28]=4.77, P=0.01$ ), whereas there was no significant difference between the HIIT and the control group. The Bax protein level in the HIIT+SAE group was significantly lower than in either the HIIT or the SAE group ( $P=0.02$ and $P<0.01$; respectively) (Fig. 2B), with no difference between the HIIT and SAE intervention groups. Furthermore, the SAE group exhibited a lower level of Bcl-2 compared to the control group $(\mathrm{F}[3,28]=4.93, P=0.05)$ (Fig. 2C), whereas the level of Bcl-2 was higher in the HIIT+SAE group vs. either the HIIT or the SAE group in tumor tissue following 4 weeks of intervention $(P<0.01$ and $P<0.01$,

A

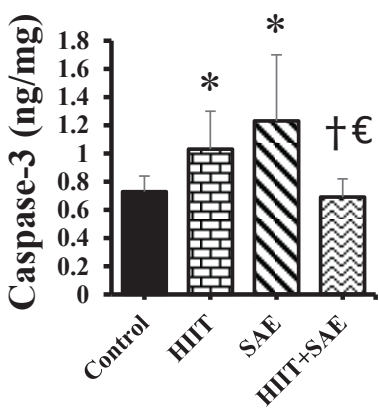

C

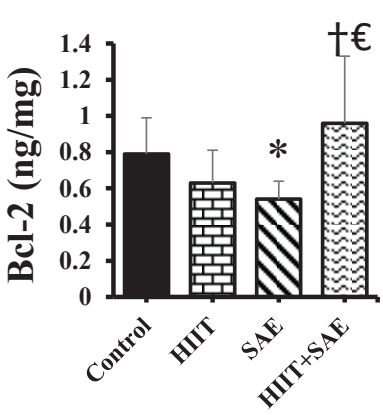

B

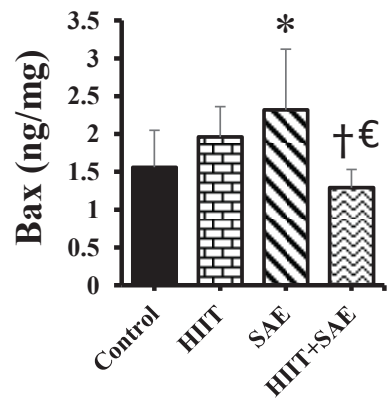

D

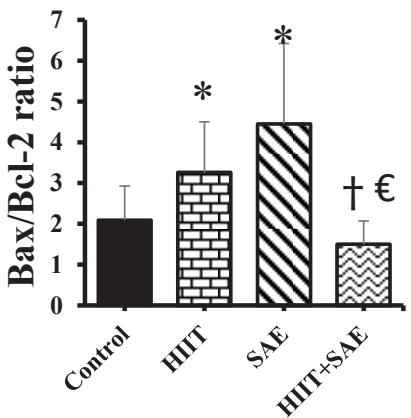

Fig. 2. Effects of HIIT and SAE on apoptosis indices in tumor tissue. (A) Caspase-3; (B) Bax; (C) Bcl-2 and (D) Bax/Bcl-2 ratio. ${ }^{\star}$ Significant difference vs. the control group; $†$ Significant difference vs. SAE group; $€$ Significant difference vs. HIIT group. HIIT: high-intensity interval training; SAE: saffron aqueous extract. 
respectively) (Fig. 2C). Finally, there was no significant difference between the HIIT and SAE groups in Bcl-2.

Additionally, both the HIIT and the SAE groups exhibited a greater ratio of Bax/Bcl-2 than the control group (F $[3,28]=8.21, P<0.01$ and $P<0.01$, respectively) (Fig. 2D). The Bax/Bcl-2 ratio in the HIIT+SAE group was significantly lower than in either the HIIT or the SAE group $(P<0.01$ and $P<0.01$, respectively), whereas no difference was found between the HIIT + SAE and the control groups.

\section{DISCUSSION}

The results of the present study showed that tumor volume was significantly smaller than in the control group following four weeks of HIIT intervention. In agreement with previous research, HIIT might delay the growth rate of breast cancer cells via its intratumoral effects [14]. Particularly, this reduced rate of tumor growth following exercise training could be due to diminished inflammation during tumor progression [14]. This finding may support the result of HIIT-mediated reduction in tumor volume, attributed to induction of apoptosis in tumor tissue in this study. Although Khori et al. [15] found that a 5-week HIIT protocol decreased Bcl-2 gene expression with increased Bax level in mice with breast cancer, this study did not observe any significant changes in these apoptotic indices in tumor tissue. However, our results demonstrated an increase in the level of caspase- 3 and in the ratio of Bax to Bcl-2 following 4 weeks of HIIT intervention, while elevated $\mathrm{Bax} / \mathrm{Bcl}-2$ ratio has been shown to up-regulate the level of caspase-3, thereby leading to apoptosis [16]. It is important to note that training intensity could elicit different and persistent growth effects through various molecular mechanisms in tumor cells of breast cancer [5]. For example, the expression of a protein, Ki-67 related with tumor cell proliferation and growth of breast cancer in rats was increased following a continuous highintensity training on the treadmill, while a low-intensity training could minimize the release of Ki-67 protein [17]. With this contradictory observation, further investigation is warranted to include the measures of tumor cell proliferation and growth (e.g., Ki-67) to gain a better understanding of HIIT-mediated apoptotic activation in breast tumor.

Furthermore, an improvement in reduced tumor volume and increased pro-apoptotic indices (caspase-3 and $\mathrm{Bax} / \mathrm{Bcl}-2$ ratio) was also observed in tumor tissue following the treatment of breast cancer with SAE supplementation. Research has shown the anti-proliferative and pro-apoptotic effects of saffron through various mechanisms [7, 18]. For example, various phytochemicals of saffron extract possess anti-inflammatory properties due to its strong antioxidant activity, which could enhance the efficacy against tumorigenesis [18]. Also, the treatment with saffron derivatives (crocin and crocetin) is associated with increased apoptosis with a reduction in blood vessels and metastasis [7]. Specifically, findings on $4 \mathrm{~T} 1$ breast cancer cells demonstrate the tumor volume reducing, anti-invasion, anti-migration and anti-adhesion properties of crocin, a component of saffron [12]. Our results may be supported by Hoshyar and colleagues [11], who showed the effect of crocin on increased $\mathrm{Bax} / \mathrm{Bcl}-2$ ratio and caspase activation in both mRNA and protein levels of human gastric adenocarcinoma cells. While the results of this study revealed positive effects of HIIT+SAE treatment on the reduction of tumor volume, no synergistic effect following both therapeutic strategies was found in apoptotic indices. Interestingly, the levels of pro- and anti-apoptotic proteins after the HIIT+SAE 
treatment were lower and higher than after either HIIT or SAE treatment in tumor tissue, respectively. Consistent with these results, Gueritat et al. [19] examined the combined effect of antioxidant supplement (pomegranate juice) and exercise training in patients with prostate cancer, but did not observe any positive effects in the expression of proliferative markers or progression of prostate cancer.

\section{CONCLUSION}

Our results indicate that either HIIT or SAE treatment alone could be effective therapeutic strategies to suppress tumor growth and/or enhance pro-apoptotic activation during tumor progression in tumor tissue of $4 \mathrm{~T} 1$ breast cancer-bearing mice. Further investigation is warranted to explore the exact anti-tumor mechanism(s) of HIIT and/or SAE, particularly associated with breast cancer.

Conflict of interest: The authors declare no conflict of interest.

\section{REFERENCES}

1. Eismann J, Heng Y, Fleischmann-Rose K, Tobias A, Phillips J, Wulf G, et al. Interdisciplinary management of transgender individuals at risk for breast cancer: case reports and review of the literature. Clin Breast Cancer 2019; 19(1).

2. Bacurau AVN, Belmonte MA, Navarro F, Moraes MR, Pontes, Jr FL, Pesquero JL, et al. Effect of a highintensity exercise training on the metabolism and function of macrophages and lymphocytes of walker 256 tumor-bearing rats. Exp Biol Med 2007; 232(10): 1289-99.

3. Dieli-Conwright CM, Lee K, Kiwata JL. Reducing the risk of breast cancer recurrence: an evaluation of the effects and mechanisms of diet and exercise. Curr Breast Cancer Rep 2016; 8(3): 139-50.

4. Jones LW, Viglianti BL, Tashjian JA, Kothadia SM, Keir ST, Freedland SJ, et al. Effect of aerobic exercise on tumor physiology in an animal model of human breast cancer. J Appl Physiol 2009; 108(2): 343-8.

5. Barra NG, Fan IY, Gillen JB, Chew M, Marcinko K, Steinberg GR, et al. High intensity interval training increases natural killer cell number and function in obese breast cancer-challenged mice and obese women. J Cancer Prev 2017; 22(4): 260.

6. Hoshyar R, Mahboob Z, Zarban A. The antioxidant and chemical properties of Berberis vulgaris and its cytotoxic effect on human breast carcinoma cells. Cytotechnology 2016; 68(4): 1207-13.

7. Lu P, Lin H, Gu Y, Li L, Guo H, Wang F, et al. Antitumor effects of crocin on human breast cancer cells. Int J Clin Exp Med 2015; 8(11): 20316.

8. Dabrowska C, Li M, Fan Y. Apoptotic caspases in promoting cancer: implications from their roles in development and tissue homeostasis. Apoptosis in Cancer Pathogenesis and Anti-cancer Therapy: Springer; 2016. pp. 89-112.

9. Liu Z, Ding Y, Ye N, Wild C, Chen H, Zhou J. Direct activation of Bax protein for cancer therapy. Med Res Rev 2016; 36(2): 313-41.

10. Pu X, Storr SJ, Zhang Y, Rakha EA, Green AR, Ellis IO, et al. Caspase-3 and caspase-8 expression in breast cancer: caspase-3 is associated with survival. Apoptosis 2017; 22(3): 357-68. 
11. Hoshyar R, Bathaie SZ, Sadeghizadeh M. Crocin triggers the apoptosis through increasing the Bax/Bcl-2 ratio and caspase activation in human gastric adenocarcinoma, AGS, cells. DNA Cell Biol 2013; 32(2): 50-7.

12. Arzi L, Farahi A, Jafarzadeh N, Riazi G, Sadeghizadeh M, Hoshyar R. Inhibitory effect of crocin on metastasis of triple-negative breast cancer by interfering with wnt $/ \beta$-catenin pathway in murine model. DNA Cell Biol 2018; 37(12): 1068-75.

13. Jensen MM, Jørgensen JT, Binderup T, Kjær A. Tumor volume in subcutaneous mouse xenografts measured by microCT is more accurate and reproducible than determined by 18 F-FDG-microPET or external caliper. BMC Med Imaging 2008; 8(1): 16.

14. Papadopoulos E, Santa Mina D. Can we HIIT cancer if we attack inflammation? Cancer Causes \& Control 2018; 29(1): 7-11.

15. Khori V, Shalamzari SA, Isanejad A, Alizadeh AM, Alizadeh S, Khodayari S, et al. Effects of exercise training together with tamoxifen in reducing mammary tumor burden in mice: possible underlying pathway of miR21. Eur J Pharmacol 2015; 765: 179-87.

16. Pawlowski J, Kraft AS. Bax-induced apoptotic cell death. Proc Natl Acad Sci 2000; 97(2): 529-31.

17. Siewierska K, Malicka I, Kobierzycki C, Paslawska U, Cegielski M, Grzegrzolka J, et al. The impact of exercise training on breast cancer. In Vivo 2018; 32(2): 249-54.

18. Siddique HR, Fatma H, Khan MA. Medicinal properties of saffron with special reference to cancer-a review of preclinical studies. Saffron: Elsevier; 2020; pp. 233-44.

19. Guéritat J, Lefeuvre-Orfila L, Vincent S, Crétual A, Ravanat J-L, Gratas-Delamarche A, et al. Exercise training combined with antioxidant supplementation prevents the antiproliferative activity of their single treatment in prostate cancer through inhibition of redox adaptation. Free Radic Biol Med 2014; 77: 95-105. 